\title{
Pinzamiento femoroacetabular tipo leva en adulto joven
}

\author{
Cam type femoroacetabular impingement in young adult \\ Luis Gerardo Domínguez Gasca, * Luis Gerardo Domínguez Carrillo \\ Citar como: Domínguez GLG, Domínguez CLG. Pinzamiento femoroacetabular tipo leva en adulto joven. \\ Acta Med Grupo Angeles. 2021; 19 (2): 298-299. https://dx.doi.org/10.35366/100462
}

Masculino de 36 años de edad, profesionista, sin antecedentes de importancia, con dolor inguinal de un año de evolución, que se exacerba posterior a juego de balompié, obligándolo a suspender la actividad. A la exploración dirigida, postura y marcha sin alteraciones, dolor a la exploración de articulación coxofemoral durante la rotación interna y aducción con flexión de cadera a $90^{\circ}$, al igual que durante abducción con cadera en posición neutra. En radiografía anteroposterior de cadera se observa anor-

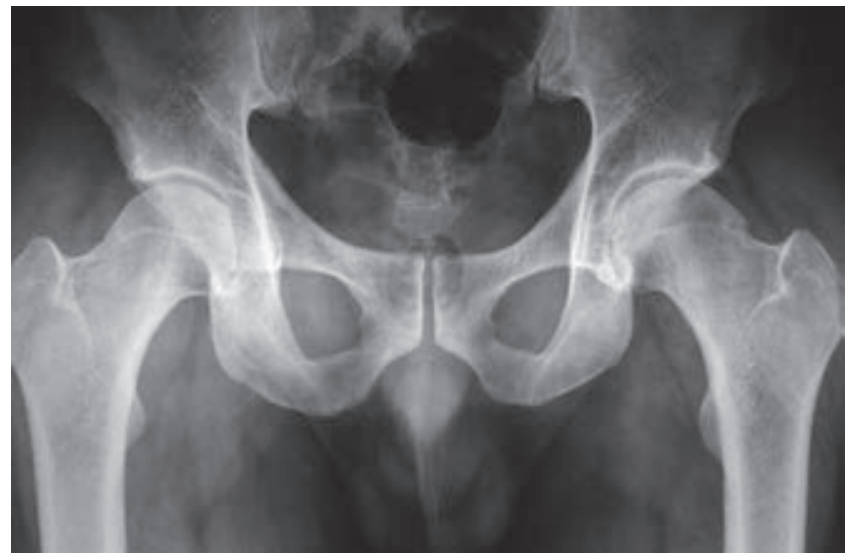

Figura 1: Radiografía anteroposterior de pelvis que muestra alteración de la unión de la cabeza con el cuello femoral izquierdo.

\footnotetext{
* Ortopedista, Cirugía Articular. División de cirugía del Hospital Ángeles León. León, Guanajuato. México.

₹ Especialista en Medicina de Rehabilitación. Catedrático de la Facultad de Medicina de León, Universidad de Guanajuato. México.
}

Correspondencia:

Dr. Luis Gerardo Domínguez Gasca

Correo electrónico: luisdom88@hotmail.com

Aceptado: 05-06-2020.

www.medigraphic.com/actamedica

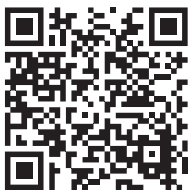

malidad de fémur izquierdo en la unión cabeza cuello, correspondiendo a pinzamiento femoroacetabular tipo leva (Figuras 1 a 3).

El pinzamiento femoroacetabular tipo leva (FAI tipo CAM por sus siglas en inglés) (la leva es un elemento mecá-
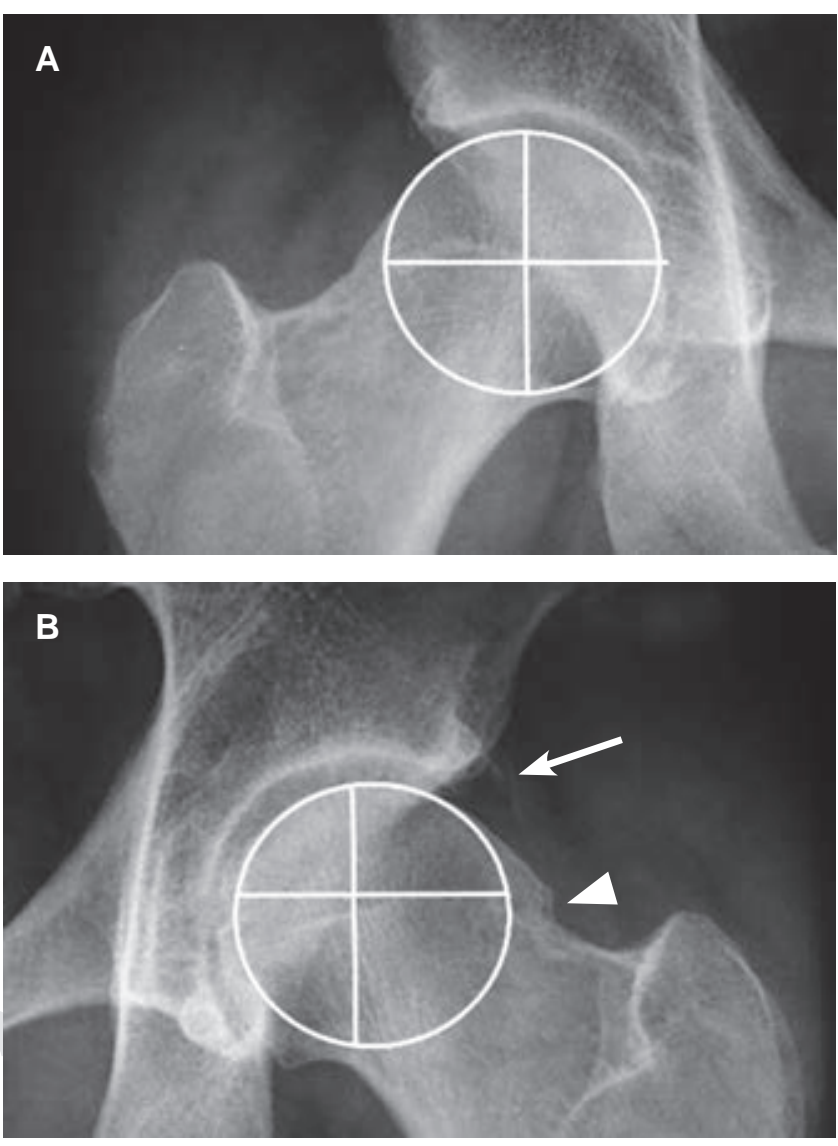

Figura 2: Acercamiento de la imagen anterior que muestra en (A) cadera derecha con eje normal de la cabeza femoral. B) Cadera izquierda con eje de rotación en un punto excéntrico a su centro geométrico que corresponde al diagnóstico de pinzamiento femoroacetabular en leva. 


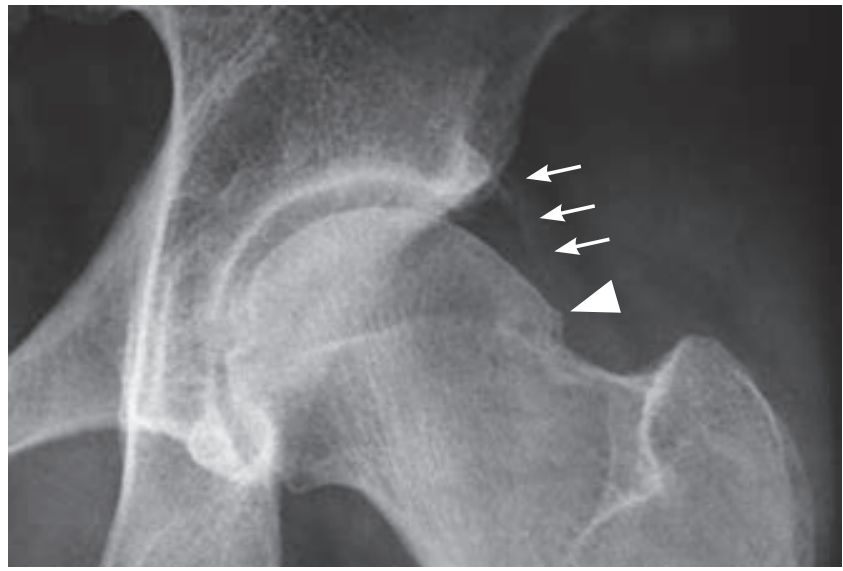

Figura 3: Imagen radiográfica con acercamiento cadera izquierda que muestra: calcificación laminar de cápsula articular en su porción externa (flechas) y osteofito en "giba" en la unión cabeza-cuello (cabeza de flecha).

nico que está sujeto a un eje en un punto que es excéntrico a su centro geométrico) es una causa conocida de dolor inguinal que puede provocar osteoartritis de la cadera. Los pacientes que padecen esta afección son principalmente jóvenes que experimentan dolor inguinal durante los movimientos de rotación interna y aducción con la articulación coxofemoral en flexión de $90^{\circ}$; ocasionado por contacto anormal entre la unión cabeza-cuello femoral y el borde acetabular debido a anormalidad anatómica del fémur, ya que la morfología de la cabeza femoral no es esférica por alteración o anomalía del cuello femoral. ${ }^{1}$ Esta anormalidad se observa en $40 \%$ de los pacientes que desarrollan osteoartritis de cadera; puede ser secundaria a enfermedades del fémur proximal típicas de la infancia, incluidas secuelas de epifisiolistesis de la cabeza femoral, enfermedad de Legg-Calvé-Perthes, a infección de la articulación coxofemoral en la infancia y traumatismos de la articulación. Al ser una patología dinámica su desarrollo también está relacionado con actividad deportiva intensa. La prevalencia de la deformidad del choque femoroacetabular tipo leva es mayor en el género masculino. ${ }^{2}$ Su diagnóstico temprano resulta importante para establecer estrategias de tratamiento en pacientes con dolor de cadera preartrítico, la serie radiográfica de cadera al igual que la resonancia magnética y la tomografía con medición del ángulo alfa proporcionan las pautas de medición prequirúrgica. ${ }^{3} \mathrm{EI}$ pinzamiento femoroacetabular se puede tratar mediante procedimiento quirúrgico abierto o por artroscopia. Se informan complicaciones clínicamente relevantes en 4.8\% de los pacientes con cirugía abierta y la tasa general de complicaciones asociadas con la artroscopia de cadera es discretamente menor (4\%). ${ }^{4}$

\section{REFERENCIAS}

1. Shanmugaraj A, Shell JR, Horner NS, Duong A, Simunovic N, Uchida $\mathrm{S}$ et al. How useful is the flexion-adduction-internal rotation test for diagnosing femoroacetabular impingement: a systematic review. Clin J Sport Med. 2020; 30 (1): 76-82. doi: 10.1097/ JSM.0000000000000575.

2. Hack K, Di Primio G, Rakhra K, Beaulé PE. Prevalence of camtype femoroacetabular impingement morphology in asymptomatic volunteers. J Bone Joint Surg Am. 2010; 92 (14): 2436-2444. doi: 10.2106/JBJS.J.01280.

3. Haldane CE, Ekhtiari S, de Sa D, Simunovic N, Ayeni OR. Preoperative physical examination and imaging of femoroacetabular impingement prior to hip arthroscopy-a systematic review. J Hip Preserv Surg. 2017; 4 (3): 201-213. doi: 10.1093/jhps/hnx020.

4. Fiorentino G, Fontanarosa A, Cepparulo R, Guardoli A, Berni L, Coviello $\mathrm{G}$ et al. Treatment of cam-type femoroacetabular impingement. Joints. 2015; 3 (2): 67-71. doi: 10.11138/jts/2015.3.2.067. 